\title{
ELECCIONES PARCIALES AL SENADO EN ALMERIA Y SEVILLA
}

Con el propósito de ocupar los escaños que dejaran vacantes Fernández Viagas (PSOE) en la provincia de Sevilla y Torres Rollón y Ponce García (ambos de UCD) en la de Almería, el día 27 de noviembre de 1980 fueron convocados a elecciones parciales al Senado los ciudadanos de ambas provincias andaluzas. Los puestos quedaron vacantes después de la dimisión, por diversos motivos, de los tres ex senadones antes mencionados.

El resultado de esta consulta electoral no iba a alterar sustancialmente la composición de la Cámara Alta, pues en ella el partido en el gobierno dispone de la mayoría absoluta de los escaños. Pero la importancia de estas elecciones viene dada por el momento histórico en el que se realizan. Se esperaba, desde todos los puntos de vista, conocer la opinión del pueblo andaluz con respecto a la política autonómica llevada a cabo por todos los partidos políticos con implantación en Andalucía. La situación política, a grandes rasgos, era la siguiente: una izquierda (PSOEPCA) que, tratando de recoger el sentimiento de rechazo generalizado en amplios sectores del pueblo andaluz con respecto al resultado del referéndum para la aplicación del artículo 151 de la Constitución, seguía reivindicando el acceso a la autonomía por la vía de este artículo. Una derecha (AP) que, en el último momento, se definió - por boca de su líder, Manuel Fraga- a favor de la aplicación del 151. $\mathrm{Y}$ un centro político (UCD) seriamente lesionado por el proceso autonómico a nivel estatal, y que, a raíz de los debates y tomas de posición durante las sesiones parlamentarias dedicadas al último voto de confianza al Gobierno, recibió el apoyo del PSA, en lo que se consideró un brusco giro en política autonómica del partido de los nacionalistas andaluces.

De otro lado existía, como ya viene siendo tristemente habitual en todo tipo de consulta electoral en nuestro país, el temor a que fuera la abstención la gran vencedora en esta convocatoria, teniendo en cuenta la tendencia a la baja en participación registrada en comicios anteriores y la fuerte abstención - al menos en el caso de Almería - habida en el referéndum para la aplicación del artículo 151 de la Constitución del 28 de febrero de 1980. 
Almería

Como ya he dicho anteriormente, eran dos los escaños a cubrir en el Senado por esta provincia. Las candidaturas fueron las siguientes:

- Por la Unión de Centro Democrático: José María Artero García, catedrático; José Moreno Pérez, funcionario judicial.

- Por el Partido Socialista Obrero Español: José Batlles Paniagua, ingeniero; Eloy José López Miralles, empleado de banca.

- Por el Partido Comunista de Andalucía: Pedro Molina García, profesor del Colegio Universitario; Aurora Claramunt Vallespí, catedrática del mismo.

- Por el Partido Socialista de Andalucía: Ramón Ponce García *, industrial; Fernando Navarrete López-Cozar, profesor del Colegio Universitario.

- Por Alianza Popular: Francisco Alados Viedma, ingeniero agrónomo; Miguel Angel Gutiérrez Ferrández, ingeniero de la Confederación Hidrográfica.

- Por Fuerza Nueva: Julián Gil de Sagredo Arribas, profesor universitario; Ginés de Haro Rossi, abogado.

- Por el Movimiento Comunista de Andalucía: Fernando Martínez López, profesor del Colegio Universitario.

- Por el Partido Liberal: Carlos Alonso Cobos, ingeniero agrónomo.

Los resultados electorales se ofrecen en el cuadro 1.

Con respecto a dicho cuadro quisiera hacer una observación: la fuente en la que me he basado para elaborarlo son los datos proporcionados por el Ministerio del Interior, y en ellos, los porcentajes obtenidos por cada uno de los candidatos han sido calculados tomando como base el número total de los votos a listas emitidos por el conjunto del electorado. Como es sabido, en esta ocasión el pueblo almeriense elegía dos senadores. No debe sorprender, por tanto, que la base a partir de la cual se han obtenido los porcentajes sea la de 243.364 votos.

Una vez aclarado este punto, y volviendo de nuevo con el cuadro 1, podemos observar cómo los dos escaños senatoriales, que anteriormente estaban en poder de UCD, han pasado a manos del PSOE, con lo que éste desplaza a UCD como fuerza hegemónica de la provincia, ya que, a partir de ahora, el PSOE contará con cinco parlamentarios - dos diputados y tres senadores - frente a los tres de UCD - dos diputados y un sólo senador.

Las dos únicas formaciones que ven incrementado su número de votantes, pese a la gran abstención, son AP y PSA. E1 PSOE, aunque gana los dos senadores, pierde 12.531 votos con relación a su candidato al Senado más votado el 1 de marzo de 1979. Lo mismo le ocurre a UCD, pero las pérdidas del partido en el gobierno casi cuadruplican a las del primer partido de la oposición: - 42.824 votos. El PCA, pese a mejorar su porcentaje, pierde 5.224 votos. Con respecto al fenómeno de la abstención haré algunas consideraciones en la parte final de este artículo.

\section{SeVilla}

En esta provincia estaba en juego la adjudicación de un escaño en el Senado: el dejado vacante por el socialista Fernández Viagas. Si en Almería fueron ocho las formaciones políticas en liza, en Sevilla se redujeron a seis, pues en esta provincia ni Fuerza Nueva ni el Partido Liberal presentaron candidatos.

Los resultados de la confrontación electoral se indican en el cuadro 2.

* Uno de los dos senadores dimitidos de UCD, posteriormente expulsados del Partido. 
CuAdro 1

CUADRO COMPARATIVO DE LOS RESULTADOS DE LAS ELECCIONES PARCIALES AL SENADO $Y$ LOS DE LAS ELECCIONES AL SENADO DEL 1-III-79 EN LA PROVINCIA DE ALMERIA

\begin{tabular}{|c|c|c|c|c|c|c|}
\hline \multicolumn{4}{|c|}{ ELECCIONES PARCIALES $27-\mathrm{XI}-80$} & \multicolumn{3}{|c|}{ ELECCIONES GENERALES 1-III-79 } \\
\hline Partido & Candidato & $\begin{array}{l}\text { Votos por } \\
\text { candidato }\end{array}$ & $\begin{array}{l}\% \text { sobre } \\
\text { suma total } \\
\text { de votos }\end{array}$ & Partido & $\begin{array}{c}\text { Votos } \\
\text { candidato } \\
\text { más votado }\end{array}$ & $\begin{array}{l}\% \text { sobre } \\
\text { suma total } \\
\text { de votos }\end{array}$ \\
\hline \multirow[t]{2}{*}{ PSOE } & José Batlles Paniagua $\ldots \ldots \ldots \ldots$ & 53.571 & 22,01 & PSOE & 66.102 & 12,78 \\
\hline & Eloy José López Miralles ... ... ... ... & 52.849 & 21,71 & & & \\
\hline \multirow[t]{2}{*}{ UCD } & 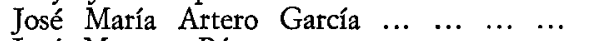 & 34.560 & 14,20 & UCD & 77.384 & 14,96 \\
\hline & 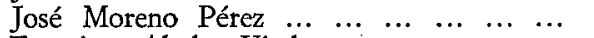 & 32.417 & 13,32 & & & \\
\hline \multirow[t]{2}{*}{ AP } & Francisco Alados Viedma $\ldots . . . .6 \ldots$ & 8.948 & 3,63 & $\mathrm{AP}(\mathrm{CD}) *$ & & \\
\hline & Miguel Angel Gutiérrez Fernández ... ... & 9.770 & 4,01 & & 9.272 & 1,79 \\
\hline \multirow[t]{2}{*}{ PCA } & M. Teresa Claramunt Vallespi $\ldots . . . .$. & 9.693 & 3,98 & PCA & & \\
\hline & 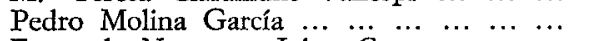 & 10.145 & 4,16 & & 15.369 & 2,97 \\
\hline \multirow[t]{2}{*}{ PSA } & Fernando Navarrete López-Cozar ... ... ... & 10.805 & 4,43 & PSA & 7.031 & 1,35 \\
\hline & 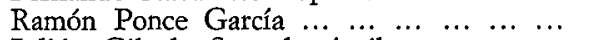 & 10.350 & 4,25 & & & \\
\hline \multirow[t]{2}{*}{ FN } & Julián Gil de Sagredo Arribas $\ldots . . .$. & 3.810 & 1,56 & FN (UN) ** & & \\
\hline & 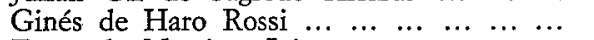 & 3.963 & 1,62 & & 4.859 & 0,93 \\
\hline \multirow{4}{*}{$\begin{array}{l}\text { MCA } \\
\text { PL }\end{array}$} & Fernando Martínez López $\ldots \ldots \ldots \ldots \ldots$ & 1.809 & 0,74 & MC-OIC *** & 2.825 & 0,54 \\
\hline & 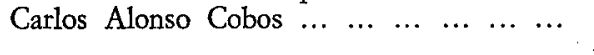 & 674 & 0,27 & $\mathrm{PL}$ & 1.976 & 0,38 \\
\hline & SUMA TOTAL vOTOS $\ldots . . . . .$. & 243.364 & 100,00 & . & & \\
\hline & 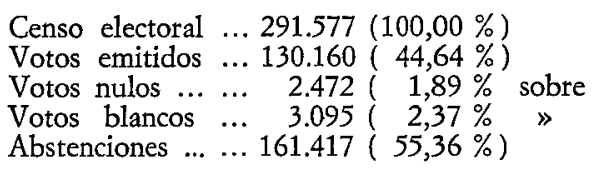 & $\begin{array}{cc}\text { total votos } \\
\gg\end{array}$ & $\begin{array}{c}\text { emitidos) } \\
\gg\end{array}$ & & & \\
\hline
\end{tabular}

* Coalición Democrática.

*** Organización de Izquierda Comunista, posteriormente fusionada con el MCA. 


\section{Cuadro 2}

CUADRO COMPARATIVO DE LOS RESULTADOS DE LAS ELECCIONES PARCIALES AL SENADO Y LOS DE LAS ELECCIONES AL SENADO DEL 1-III-79 EN LA PROVINCIA DE SEVILLA

\begin{tabular}{|c|c|c|c|c|c|c|}
\hline \multicolumn{4}{|c|}{ ELECCIONES PARCIALES 27-XI-80 } & \multicolumn{3}{|c|}{ ELECCIONES GENERALES 1-III-79 } \\
\hline Partido & Candidato & $\begin{array}{l}\text { Votos por } \\
\text { candidato }\end{array}$ & $\begin{array}{l}\% \text { sobre } \\
\text { suma total } \\
\text { de votos }\end{array}$ & Partido & $\begin{array}{l}\text { Votos } \\
\text { candidato } \\
\text { más votado }\end{array}$ & $\begin{array}{l}\% \text { sobre } \\
\text { suma total } \\
\text { de votos }\end{array}$ \\
\hline $\begin{array}{l}\text { PSOE } \\
\text { UCD } \\
\text { PCA } \\
\text { AP } \\
\text { PSA } \\
\text { MCA }\end{array}$ & 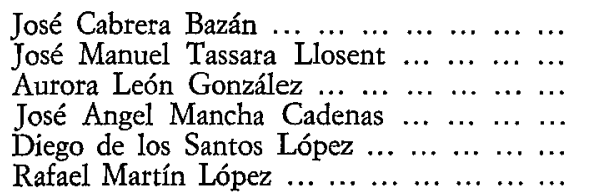 & $\begin{array}{r}192.224 \\
36.395 \\
95.632 \\
37.193 \\
51.796 \\
2.647\end{array}$ & $\begin{array}{r}46,22 \\
8,75 \\
23,00 \\
8,94 \\
12,45 \\
0,64\end{array}$ & $\begin{array}{l}\text { PSOE } \\
\text { UCD } \\
\text { PCA } \\
\text { AP (CD) * } \\
\text { PSA } \\
\text { MC-OIC ** }\end{array}$ & $\begin{array}{r}204.893 \\
174.453 \\
114.544 \\
34.129 \\
115.601 \\
1.745\end{array}$ & $\begin{array}{r}10,32 \\
8,78 \\
5,76 \\
1,71 \\
5,82 \\
0,08\end{array}$ \\
\hline & 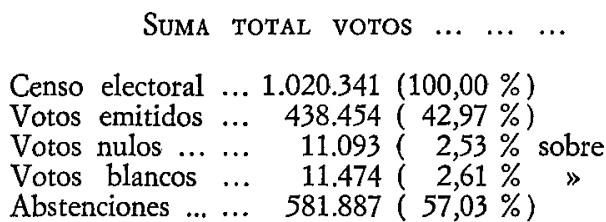 & $\begin{array}{c}\text { total votos } \\
\gg \quad \gg\end{array}$ & $\begin{array}{c}\text { emitidos) } \\
\gg\end{array}$ & & & \\
\hline
\end{tabular}

* Coalición Democrática.

** Organización de Izquierda Comunista, posteriormente fusionada con el MCA.

FUENTE: Ministerio del Interior. 
A la vista de los datos del cuadro 2, resulta evidente que el PSOE conserva su escaño senatorial en la provincia, mejorando porcentajes, pero perdiendo 12.669 votos. Muy pocos votos pierden los socialistas sevillanos, si comparamos estas pérdidas con las de UCD, que en esta ocasión, y comparando sus resultados con los de su candidato más votado - por supuesto en el Senado- en las generales de marzo de 1979, arrojan un saldo de - 138.058 votos. Este es uno de los más grandes reveses electorales que sufre el partido en el gobierno, pues, además, se ve relegado al quinto puesto - inmediatamente por delante del MCA, que es el partido menos votado- en una provincia de la importancia de Sevilla. Otro partido que sale seriamente dañado de las elecciones parciales en esta provincia es el PSA, pues pierde la importante cantidad de 63.805 votos. El PCA, que supera ampliamente sus porcentajes anteriores, pierde 18.912 votos, erigiéndose en la segunda fuerza política de la provincia. Los dos únicos partidos que ven aumentado su número de seguidores son AP y el MCA, a pesar de la gran abstención registrada.

\section{Abstención}

Ofrezco a continuación el cuadro 3, donde puede apreciarse con claridad la evolución de la abstención desde el Referéndum para la Reforma Política de 1976 hasta las elecciones parciales que estamos comentando.

Comparando los índices de participación de ambas provincias, comprobamos que, si bien Almería comenzó la andadura democrática con mayor índice de participación que Sevilla, en las siguientes consultas registra más altas cotas de abstención ciudadana y siempre en un sentido creciente.

En la provincia sevillana, sin embargo, habiéndose llegado al 36 por 100 de abstención en las elecciones municipales de 1979, en la consulta siguiente, el referéndum para la aplicación del artículo 151 de la Constitución, se registran nueve puntos más en cuanto a participación, rebajándose la cota de abstención al 27,6 por 100. Pero con respecto a este último referéndum, y en la misma provincia, impresiona comprobar que la abstención ha aumentado un 30 por 100 (el 27,06 por 100 en el referéndum para la aplicación del artículo 151 y el 57,03 por 100 en las elecciones parciales al Senado).

\section{CoNCLUSIón}

Un cualificado miembro del PSOE ha calificado los resultados de estas parciales como «indicativos del cambio que se ha dado en el electorado del país». Personalmente creo que, efectivamente, denotan un cambio de rumbo, pero que, sólo en este caso, va en beneficio del primer partido de la oposición. Me explico: recientemente se han celebrado elecciones a los Parlamentos vasco y catalán. En los dos casos ha habido un claro retroceso en la aceptación del Partido Centrista, pero este retroceso no ha jugado en favor del PSOE en ninguno de los dos casos, pues los grandes beneficiados han sido los grupos de marcado carácter nacionalista. En el caso que nos ocupa, los errores del único partido de tendencia nacionalista -el PSA-, unidos, es cierto, al mantenimiento de una política coherente y sin variaciones por parte de la izquierda (PSOE-PCA), en una región en la que ésta goza de una mayoría indiscutible, han sido los factores que han producido el derrumbamiento centrista y nacionalista en beneficio de la izquierda, y, dentro de ésta, y al entrar en juego la idea del voto útil o «más útil», al PSOE. 


\section{CUADRO 3}

CUADRO COMPARATIVO DE LA PARTICIPACION POPULAR EN LAS PROVINCIAS DE ALMERIA Y SEVILLA EN LOS DISTINTOS PROCESOS ELECTORALES

\begin{tabular}{|c|c|c|c|c|c|c|c|}
\hline Provincias & $\begin{array}{l}\text { Referéndum } \\
\text { reforma política } \\
1976(\%)\end{array}$ & $\begin{array}{l}\text { Elecciones } \\
\text { generales } \\
1977(\%)\end{array}$ & $\begin{array}{c}\text { Referéndum } \\
\text { constitucional } \\
1978(\%)\end{array}$ & $\begin{array}{l}\text { Elecciones } \\
\text { generales } \\
1979(\%)\end{array}$ & $\begin{array}{l}\text { Elecciones } \\
\text { locales } \\
1979(\%)\end{array}$ & $\begin{array}{c}\text { Referéndum } \\
\text { aplicación } \\
\text { art. 151 Const. } \\
1980 \text { (\%) }\end{array}$ & $\begin{array}{c}\text { Elecciones } \\
\text { parciales } \\
\text { Senado } \\
1980(\%)\end{array}$ \\
\hline \multicolumn{8}{|l|}{ Almería } \\
\hline $\begin{array}{llllll}\text { Participación } & \ldots & \ldots & \ldots & \ldots\end{array}$ & 81,7 & 76,0 & 67,1 & 66,7 & 58,0 & 51,01 & 44,64 \\
\hline $\begin{array}{lllll}\text { Abstención } & \ldots & \ldots & \ldots & \ldots\end{array}$ & 18,3 & 24,0 & 32,9 & 33,3 & 42,0 & 48,98 & 55,36 \\
\hline \multicolumn{8}{|l|}{ Sevilla } \\
\hline $\begin{array}{lllll}\text { Participación } & \ldots & \ldots & \ldots & \ldots\end{array}$ & 80,5 & 76,0 & 72,3 & 71,5 & 64,0 & 72,93 & 42,97 \\
\hline $\begin{array}{lllll}\text { Abstención } & \ldots & \ldots & \ldots & \ldots\end{array}$ & 19,5 & 24,0 & 27,7 & 28,5 & 36,0 & 27,06 & 57,03 \\
\hline
\end{tabular}

FuENTE: Ministerio del Interior. 
Para terminar, y en mi opinión, el fantasma del 28 de febrero ha estado presente en el ánimo de los electores andaluces a la hora del rechazo a la política de UCD, rechazo que no sólo ha rozado, sino que ha alcanzado de lleno al PSA por sus últimas coincidencias en el tema autonómico con el Partido Centrista. 